\title{
Novel Predictive Markers on Computed Tomography for Predicting Early Epidural Hematoma Growth in Pediatric Patients
}

\author{
Avery A. Kopacz ${ }^{1}$ Hunter Miears ${ }^{1}$ Reagan A. Collins ${ }^{1}$ Laszlo Nagy $^{1}$ \\ ${ }^{1}$ Department of Pediatrics, Texas Tech University Health Sciences \\ Center, Lubbock, Texas, United States \\ Address for correspondence Avery A. Kopacz, BS, Department of \\ Pediatrics, Texas Tech University Health Sciences Center, TTUHSC \\ J Neurosci Rural Pract 2021;12:689-693. \\ SOM, 3601 4th Street, Lubbock, TX 79415, United States \\ (e-mail: avery.kopacz@ttuhsc.edu).
}

\section{Abstract \\ Keywords \\ - Epidural Hematoma \\ - CT Blend Sign \\ - CT Swirl Sign \\ - CT Black Hole Sign \\ - Intracranial Hemorrhage}

Objective Epidural hematoma $(E D H)$, most often caused by rupture of the middle meningeal artery secondary to head trauma with fracture of the temporal bone, is a potentially fatal condition that can lead to elevated intracranial pressure, herniation, and death within hours following the inciting traumatic incident, unless surgical evacuation is accomplished. Several markers have been found to be associated with hematoma expansion in intracerebral hemorrhage (ICH) patients, including: the CT Blend Sign, Swirl Sign, and Black Hole Sign. This study aims to examine these markers, along with intradural air close to or in the region of an EDH and/or close to a significant fracture, fractures involving the skull base, and complicated (i.e., comminuted or displaced) fractures for possible associations to EDH growth in the pediatric population. Predicting hematoma growth is a crucial part of patient management, as surgery can be a life-saving intervention. Methods Scans from all pediatric patients with EDH from 2012 to 2019 across two separate health systems were examined and measurements were taken to determine whether these additional factors are of predictive value. Specifications such as length, transverse, and height measurements were taken from CT images.

Statistical Analysis The average percent change in the hematoma measurements was used to determine which predictive factors were associated with a "noteworthy increase," namely, an increase of greater than $25 \%$. Additionally, the average percent change in hematoma size was evaluated for patients whose original imaging showed either all three CT signs or intradural air in all three specified locations.

Results Most of the proposed markers were associated with EDH growth in this cohort. The established CT signs were also supported. This is notable, as most of the research on these signs has been in adult populations rather than pediatric.

Conclusions Adding these novel imaging signs could aid in the decision to operate on versus observe PEDH patients, thereby preventing unnecessary procedures or preserving brain function quickly when surgery is indicated. This study serves as a starting point for several other investigations into the validity of the proposed markers as well as a reevaluation of the current signs in the pediatric population. published online September 28, 2021
DOI https://doi.org/

10.1055/s-0041-1735327. ISSN 0976-3147. (c) 2021. Association for Helping Neurosurgical Sick People. All rights reserved.

This is an open access article published by Thieme under the terms of the Creative Commons Attribution-NonDerivative-NonCommercial-License, permitting copying and reproduction so long as the original work is given appropriate credit. Contents may not be used for commercial purposes, or adapted, remixed, transformed or built upon. (https://creativecommons.org/ licenses/by-nc-nd/4.0/)

Thieme Medical and Scientific Publishers Pvt. Ltd., A-12, 2nd Floor, Sector 2, Noida-201301 UP, India 


\section{Introduction}

Epidural hematoma (EDH), most often caused by rupture of the middle meningeal artery secondary to head trauma with fracture of the temporal bone, is a potentially fatal condition that can lead to elevated intracranial pressure (ICP), herniation, and death within hours following the inciting traumatic incidence, unless surgical evacuation is accomplished ${ }^{1,2}$ (-Fig. 1A). Fractures of the calvarium can also contribute venous blood to the hematoma from the damaged diploë. ${ }^{3}$ Subacute and chronic forms of EDH have also been documented. ${ }^{4-6}$ In fact, with the standardization of repeat CT scans for patients with head injury, these forms of progressive epidural hematoma (PEDH) have been found to be more common than previously expected, occurring in approximately $30 \%$ to $42.3 \%$ of head trauma patients. ${ }^{7-10}$ Development of PEDH often occurs within 3 days postinjury but can extend up to 11 days in rare cases. ${ }^{10,11}$ Since PEDH patients are likely to exhibit elevated ICP and require craniectomy for hematoma removal, in order to prevent the potentially fatal sequelae seen in acute cases, a reliable predictor of hematoma growth would be useful to guide early and appropriate intervention. ${ }^{10}$

Several markers have been identified as potential predictors of early hematoma growth in intracerebral hemorrhage
(ICH) patients, including the CT Black Hole Sign, the CT Blend Sign, and the CT Swirl Sign. ${ }^{3,11,12}$ The CT Black Hole Sign presents as foci of hypo- or isoattenuation within the hematoma and indicates heterogenicity of the hematoma, which has been associated with hematoma growth and poor outcomes $^{11}$ (-Fig. 1B). The CT Blend Sign signifies a heterogeneous hematoma made up of blood with mixed CT densities which may occur due to liquid blood secondary to active bleeding $^{3}$ (-Fig. 1C). Similarly, the CT Swirl Sign appears as a mixed-density hematoma, which is found to be associated with active bleeding as a result of EDH ( - Fig. 1D). Kim et al have found that this sign denotes a heterogeneous hematoma, which correlates with a larger hematoma volume and a higher mortality rate, although it was not independently and specifically predictive of hematoma growth. ${ }^{12,13}$ Both variables have been found to be closely associated with the CT Blend Sign and early hematoma growth. The CTA Spot Sign is also referenced often in the literature but is used for ICH, rather than epidural, so was not included in this study. ${ }^{14}$ Although head injury in children and young adults is the leading cause of death in this age group, these imaging markers are mostly reported in adult populations. ${ }^{3,11,12,15}$

The specific aim of this study is to evaluate and to compare the utility of imaging markers, including the CT Blend Sign,

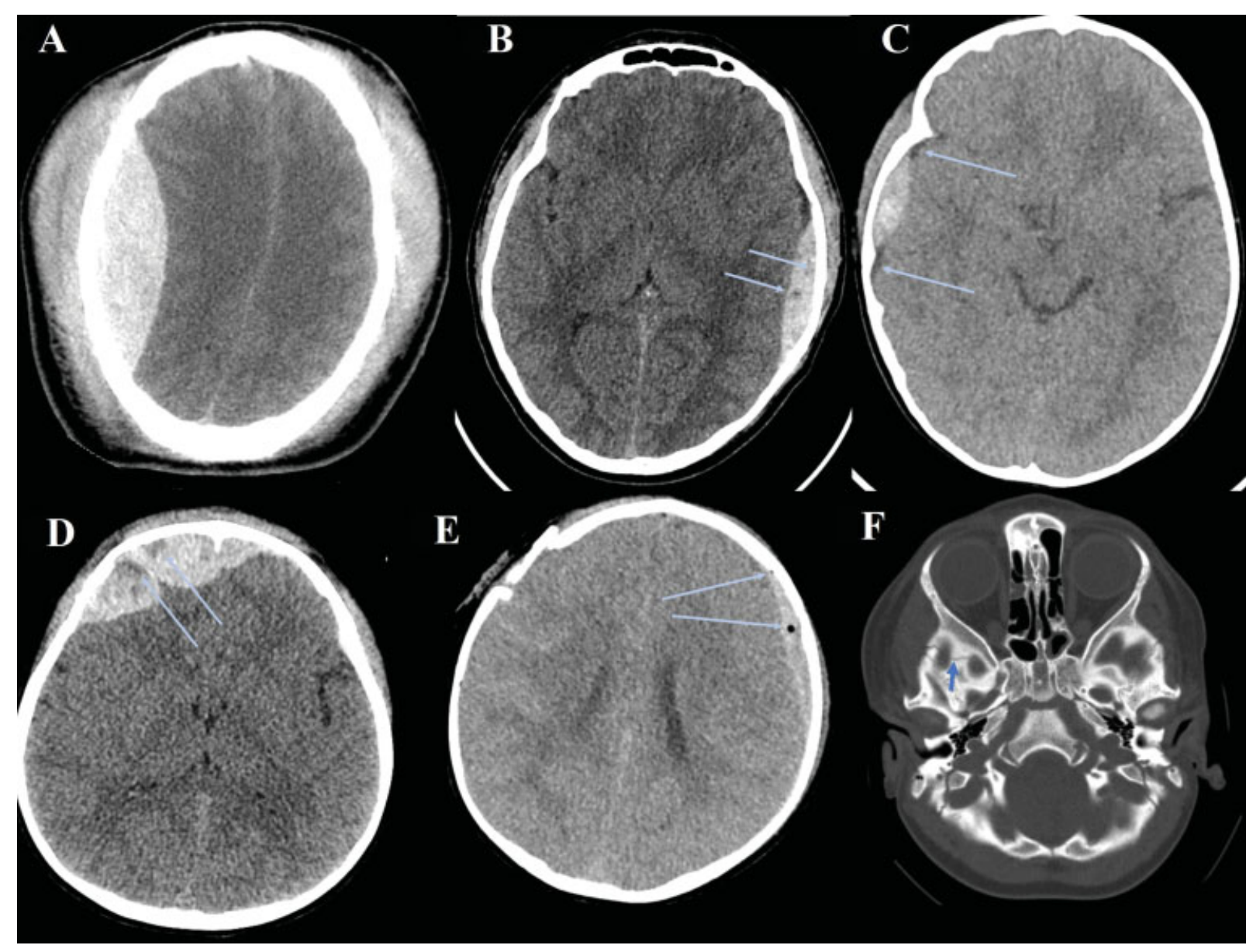

Fig. 1 (A) Right parietal epidural hematoma (EDH); axial noncontrast Computed tomography (CT). (B) Left parietal EDH with CT Black Hole Sign (arrows); axial noncontrast CT. (C) Right parietal EDH with CT Blend Sign (arrows); axial noncontrast CT. (D) Right frontal EDH with CT Swirl Sign (arrows); axial noncontrast CT. (E) Left frontoparietal EDH with intradural air (arrows); right frontal comminuted and displaced fracture; axial noncontrast CT. (F) Fracture of the skull base (arrows); axial bone window CT. 
Black Hole Sign, and Swirl Sign, as well as some novel markers, in predicting PEDH growth in the pediatric population. Although not yet supported by the current literature, some additional markers will be specifically examined in this study for their association with hematoma expansion: intradural air close to or in the region of an EDH and/or close to a significant fracture, fracture involving the skull base, and complicated (i.e., comminuted or displaced) fractures (-Fig. 1E, F).

Aside from the predictive power of the imaging markers, practical application of these in the clinical setting should be considered, as this also determines their utility as a predictor of hematoma growth, specifically in children less than 18 years of age.

\section{Methods}

Selection criteria for this study included all pediatric patients ( $<18$ years of age) presenting with $\mathrm{EDH}$, who underwent a baseline CT scan within 6 hours after hospital admission or presentation of symptoms, whichever occurred first, and a follow-up CT scan within 24 hours of baseline imaging between January 1, 2012, and January 1, 2019. This patient list was generated using the relevant ICD9 and ICD10 codes for EDH. Please note that the study population includes all pediatric patients with EDH, not just PEDH. Information regarding patient demographics, type and location of injury, and measurements of the hematomas for each CT was taken from the electronic health records of two separate hospital systems. Patient CT scans were examined by two examiners, and information about the size and location of the hematomas and the presence or absence of the abovementioned markers were recorded using the internal measurement feature in the imaging software. Specific measurements included length (anteroposterior), transverse, height (craniocaudal), and volume in centimeters and $\mathrm{cm}^{3}$.

Pre- and postdifferences in length (anteroposterior), transverse, height (craniocaudal), and volume were obtained using Wilcoxon signed rank test. The nonparametric independent samples Mann-Whitney U test was used to determine the differences in various predictors for differences in length, transverse, height, and volume. Time and type of intervention were also analyzed for potential benefits of early and appropriate intervention on patient outcomes.

Additionally, the percent changes in length, transverse, height, and volume measurements were calculated and compared among patients who had any or all of the literature-supported and proposed markers.

\section{Results}

A total of 116 patient charts were reviewed with encounters from 2012 to 2019 across two separate health systems. Of these, 25 patients were identified as fitting the study criteria, with the most common exclusionary reason being that many patients had an initial CT but their follow-up imaging was a MRI scan. When this was the case, the authors did not feel
Table 1 Average percent change in length, transverse, and height measurements of all hematomas

\begin{tabular}{|l|l|l|l|l|}
\hline Overall & Length & Transverse & Height & Volume \\
\hline Mean percent & $15 \%$ & $79 \%$ & $66 \%$ & $182 \%$ \\
change & \pm 0.38 & \pm 2.6 & \pm 0.58 & \pm 0.033 \\
\hline
\end{tabular}

Note: $n=25$.

Table 2 Statistical significance of proposed fracture markers; significance set to $p<0.05$

\begin{tabular}{|l|l|l|}
\hline Parameter & $p$-Value & Significance \\
\hline $\begin{array}{l}\text { Fracture involving the } \\
\text { skull base a }\end{array}$ & $\mathbf{0 . 0 1 4}$ & Significant \\
\hline Comminuted fracture $^{\mathrm{a}}$ & $\mathbf{0 . 0 3 3}$ & Significant \\
\hline Displaced fracture $^{\mathrm{b}}$ & 0.748 & Not significant \\
\hline
\end{tabular}

Note: Bold values are significant at $p<0.05$.

${ }^{a}$ Specifically the transverse measurement of the hematoma.

bspecifically the length measurement of the hematoma.

comfortable making statistical claims regarding any changes in hematoma size between the two different imaging modalities. The average time between the initial and follow-up CT scans was 4.9 hours. The average percent change ([follow up measurement - initial measurement] / initial measurement $\times 100 \%$ ) in each of the hematoma measurements is shown in -Table 1.

Of the proposed fracture markers, only patients with a fracture of the skull base and/or comminuted fractures showed a significant increase in hematoma size as shown in - Table 2.

Of the 25 patients, those who had a greater than $25 \%$ increase in any of the hematoma measurements were evaluated with respect to each marker as shown in - Table 3. It was found that over $50 \%$ of these patients had a "noteworthy increase" (greater than $25 \%$ as per the study protocol) in both length, height, and volume measurements of their hematomas for each of the proposed air markers. In other words, more than $50 \%$ of patients with air in any of the three specified regions had a noteworthy increase in the length, height, and volume of their hematoma. Most compelling is the high percentages of patients who had both air in any of the three regions and a notable increase in hematoma volume ( $80 \%, 79 \%$, and $77 \%$, respectively). Additionally, $50 \%$ of patients with either Blend, Swirl, or Black Hole signs had a greater than $25 \%$ increase in the height measurement of their hematoma.

It was hypothesized that the presence of air in all three locations or the presence of all three CT signs could be correlated to increased severity of the hematoma and therefore greater risk of hematoma growth. Thus, the average percent change in hematoma measurement was evaluated by the number of patients who had air in all three locations (11 out of 25) or all three CT signs ( 9 out of 25). The results of which are listed in - Table 4. 
692 Novel Predictive Markers for Predicting Early Epidural Hematoma Growth Kopacz et al.

Table 3 Patients with significant (> 25\%) increase in hematoma size by marker and measurement

\begin{tabular}{|c|c|c|c|c|}
\hline & Length & Transverse & Height & Volume \\
\hline Intradural air in the region of hematoma on CT & $71 \%$ & $38 \%$ & $63 \%$ & $80 \%$ \\
\hline Intradural air in the region of fracture on CT & $57 \%$ & $38 \%$ & $56 \%$ & $79 \%$ \\
\hline Intradural air in the region of skull base & $71 \%$ & $38 \%$ & $56 \%$ & $77 \%$ \\
\hline CT Blend Sign & $43 \%$ & $38 \%$ & $50 \%$ & $42 \%$ \\
\hline CT Swirl Sign & $43 \%$ & $25 \%$ & $50 \%$ & $71 \%$ \\
\hline CT Black Hole Sign & $43 \%$ & $50 \%$ & $50 \%$ & $59 \%$ \\
\hline Total patients with percent change $>25 \%$ & $7 / 25$ & $8 / 25$ & $16 / 25$ & $19 / 25$ \\
\hline
\end{tabular}

Note: Bold values represent significance.

Table 4 Percent change in hematoma size in patients with all three air markers or all three CT signs

\begin{tabular}{|l|l|l|l|l|l|}
\hline Average \% increase & Length & Transverse & Height & Volume & $n /$ Total \\
\hline All 3 air signs & $22 \%$ & $27 \%$ & $55 \%$ & $83 \%$ \\
& \pm 0.43 & \pm 0.41 & 0.51 & 0.89 & $11 / 25$ \\
& $2 \%$ & $140 \%$ & $37 \%$ & $218 \%$ & $9 / 25$ \\
& \pm 0.23 & \pm 4.3 & \pm 0.60 & 4.84 & \\
\hline
\end{tabular}

\section{Discussion}

The small sample size in this study imposes some limitations on the interpretation of the data collected. However, the high percentage of patients with intradural air who had noteworthy hematoma expansion represents a promising new predictive marker. This could be explained by a similar mechanism to the skull base and complicated fractures; more severe trauma could introduce air into the calvarium, associated with greater potential for damage to the meningeal vasculature and diploic vessels, which causes increased blood extravasation into the epidural space. Due to the sharply defined borders and contrast, identifying air in a scan is not difficult and could provide rapid identification of growth risk, thus aiding in the decision of whether or not to operate on a patient earlier in their treatment course.

Additionally, the demonstrated predictive potential of skull base and comminuted fractures shows another potentially useful new marker that could be helpful in predicting the propensity of a PEDH to grow. The relationship between the presence of complicated fractures and hematoma growth is intuitive, since a more extensive fracture would likely be associated with more traumatic head injuries, resulting in increased blood extravasation as discussed above. While both of the abovementioned imaging features may seem to be obviously connected to hematoma growth, the utility of this study lies in the fact that their predictive value has not actually been established in the literature until now.

While the CT Blend, Swirl, and Black Hole signs did show a relationship between presence on initial scan and hematoma growth, these signs were not present as often as intradural air in patients with significant growth. Additionally, there is more room for interpretation when determining if these markers are present on imaging. These signs are discussed extensively in the literature and were used largely as a type of control with which to compare the proposed markers. Thus, using the presence of intradural air as a possibly more sensitive, rather than specific predictor of hematoma growth in conjunction with the established Blend, Swirl, and Black Hole signs may be beneficial. Further investigation regarding patient outcomes in this scenario is required.

This study should serve as a starting point for several more investigations, preferably with a far larger cohort, to assess the validity of these results. It could also be useful to examine the possible use of intradural air as a predictive marker for hematoma growth in the adult population. Ascertaining if there are particular combinations of Blend, Swirl, and Black Hole Signs with intradural air correlated to greater hematoma growth might also be of interest. Additionally, further studies assessing the validity of skull base and other associated fractures could be helpful in solidifying the predictive value of these markers in assessing PEDH growth. Although the results of this study do not warrant radical changes to current protocols, the value lies in the possibility for further research into an important factor in the diagnosis and management of PEDH patients, as well as the addition of another tool to aid in clinical decision-making with regard to whether or not to send a patient to surgery. Catching growing hematomas early can hopefully decrease the morbidity and mortality often associated with this condition.

\section{Conclusion}

The presence of intradural air and CT Blend, Swirl, and Black Hole signs were found in over half of the patients with progressive hematoma growth in this group. The complexity of the involved fracture (involvement of skull base and 
comminuted status) appeared to be a significant determinant of EDH growth in this cohort. Due to the easily recognizable characteristics of intradural air and the high percentage of patients in this cohort with this feature on $\mathrm{CT}$, it would be reasonable to use intradural air as a predictive tool for hematoma growth. Therefore, it may prove beneficial to a patient with these imaging features to evacuate the hematoma rather than putting them under observation. Given the sample size and restriction to pediatric patients, the authors hope to expand the cohort to include adult patients and further test the predictive value of these proposed parameters. Establishing such new markers can hopefully provide more effective care for patients with EDH.

Note

This study was reviewed and approved by the International Review Board, Approval \#L18-148.

\section{Authors' Contributions}

A.K.: protocol, data collection, and manuscript review;. H.M.: data collection and manuscript review; R.C.: statistical support and manuscript review; and L.N.: supervisory support and manuscript review.

\section{Conflict of Interest}

None declared.

\section{References}

1 Zussman BM, Goldschmidt E, Faraji AH, Salvetti DJ, Jankowitz BT. Middle meningeal artery embolization for the treatment of an expanding epidural hematoma. World Neurosurg 2019; 128:284-286

2 Al-Mamoori MJ. Management of epidural hematoma in the pediatric age group. Med J Babylon 2019;16:276-285

3 Li Q Zhang G, Huang YJ, et al. Blend Sign on computed tomography: novel and reliable predictor for early hematoma growth in patients with intracerebral hemorrhage. Stroke 2015;46(08): 2119-2123

4 Liu Y, Wang Y, Song T, et al. Chronic epidural haematoma: a report of 10 cases and review of the literature. J Clin Neurosci 1999;6 (05):412-415

5 Stein SC, Spettell C, Young G, Ross SE. Delayed and progressive brain injury in closed-head trauma: radiological demonstration. Neurosurgery 1993;32(01):25-30, discussion 30-31

6 Chang EF, Meeker M, Holland MC. Acute traumatic intraparenchymal hemorrhage: risk factors for progression in the early postinjury period. Neurosurgery 2006;58(04):647-656, discussion 647-656

7 Tong WS, Zheng P, Xu JF, et al. Early CT signs of progressive hemorrhagic injury following acute traumatic brain injury. Neuroradiology 2011;53(05):305-309

8 Chen H, Guo Y, Chen SW, et al. Progressive epidural hematoma in patients with head trauma: incidence, outcome, and risk factors. Emerg Med Int 2012;2012:134905

9 Borovich B, Braun J, Guilburd JN, et al. Delayed onset of traumatic extradural hematoma. J Neurosurg 1985;63(01):30-34

10 Boulouis G, van Etten ES, Charidimou A, et al. Association of key magnetic resonance imaging markers of cerebral small vessel disease with hematoma volume and expansion in patients with lobar and deep intracerebral hemorrhage. JAMA Neurol 2016;73 (12):1440-1447

11 Li Q Zhang G, Xiong X, et al. Black hole sign: novel imaging marker that predicts hematoma growth in patients with intracerebral hemorrhage. Stroke 2016;47(07):1777-1781

12 Kim J, Smith A, Hemphill JC III, et al. Contrast extravasation on CT predicts mortality in primary intracerebral hemorrhage. AJNR Am J Neuroradiol 2008;29(03):520-525

13 Pruthi N, Balasubramaniam A, Chandramouli BA, et al. Mixeddensity extradural hematomas on computed tomography-prognostic significance. Surg Neurol 2009;71(02):202-206

14 Wada R, Aviv RI, Fox AJ, et al. CT angiography “spot sign" predicts hematoma expansion in acute intracerebral hemorrhage. Stroke 2007;38(04):1257-1262

15 Maugeri R, Anderson DG, Graziano F, Meccio F, Visocchi M, Iacopino DG. Conservative vs. surgical management of posttraumatic epidural hematoma: a case and review of literature. Am J Case Rep 2015;16:811-817 